\title{
5-Hydroxytryptamine attenuates free radical injury in primary mouse cortical cultures
}

\author{
Jee Youn Kang,' Hyo Jung Kang,' Young Ki Chung,,3 Byoung Joo Gwag, ${ }^{2,3}$ and Jai Sung Noh 1,3,CA
}

\author{
Departments of 'Psychiatry and Behavioral Sciences, ${ }^{2}$ Pharmacology, and ${ }^{3}$ Brain Disease Research Center, Ajou University, \\ School of Medicine, Suwon, Kyungkido 442-749, Korea \\ CA,I Corresponding Author and Address
}

Received 2I December 2000; accepted 24 January 200 I

\begin{abstract}
The effects of 5-hydroxytryptamine (5-HT) on several types of neuronal injury in mouse cortical cell cultures were tested. Co-treatment with 5-HT prevented free radical-mediated neuronal necrosis induced by $\mathrm{FeCl}_{2}$ or buthionine sulfoximine (BSO) in a dose-dependent manner. Subtype antagonists did not reverse the protective effect and 5-HT showed direct free radical scavenging activity evidenced by its ability to reduce the
\end{abstract}

stable free radical I,I-diphenyl-2-picrylhydrazyl (DPPH) in a cell-free system. Excitotoxic necrosis induced by NMDA or apoptosis induced by staurosporine was not sensitive to 5-HT treatment. These features raise the possibility that the endogenous neurotransmitter 5-HT may work as an innate antioxidant defense mechanism in the CNS. NeuroReport 12:963-966 (C) 200I Lippincott Williams \& Wilkins.

Key words: Aapoptosis; Cortical cultures; Free radicals; 5-Hydroxytryptamine; Necrosis

\section{INTRODUCTION}

An abundance of evidence shows that endogenous neurotransmitters exert a modulating role in neurodegeneration through pharmacological or chemical pathways. As an example, activation of different subtypes of receptors in the glutamatergic system can cause or dampen neuronal injury [1-3]. Dopamine also has dual effects on neurodegeneration. For example, in high concentration, dopamine can cause apoptosis of neurons through the formation of conjugates with the cysteine residues of protein, but in low concentration, it can prevent neuronal injury induced by free radicals [4].

5-Hydroxytryptamine (5-HT), a monoamine neurotransmitter, is synthesized from tryptophan by tryptophan hydroxylase in serotonergic neurons of mesencephalon and distributed diffusely to other brain areas, especially neocortex, striatum and hypothalamus [5]. Also 5-HT plays a significant role in many higher cortical functions and its dysfunction has been proposed as a cause of psychotic states, mood changes and/or sleep abnormalities [6]. Recently, considerable evidence suggests that $5-\mathrm{HT}$ or its receptor agonists affect neuronal injury. For example, 5-HT increases neuronal survival or prevents neuronal injury in cultures [7,8]. Selective $5-\mathrm{HT}_{1 \mathrm{~A}}$ receptor agonists prevent neuronal damage in vivo and in vitro [9-13]. In contrast, 5HT produces apoptotic neuronal injury through oxidative metabolism [14].

To clarify the apparent contradictions in action of 5-HT on neuronal injury, we set out to investigate the effects of 5-HT on three well defined death paradigms, namely oxidative neuronal necrosis, excitotoxic necrosis and staurosporine-induced neuronal apoptosis in cortical cultures.

Here we report that 5-HT attenuates free radical-induced neuronal injury through radical scavenging activity without affecting excitotixcity or stausporine-induced apoptosis in mouse cortical cell cultures.

\section{MATERIALS AND METHODS}

Cell cultures: Cortical cell cultures were prepared as described previously [15]. In brief, dissociated cortical cells obtained from fetal mice (E14) were plated onto 24-well plates precoated with $100 \mu \mathrm{g} / \mathrm{ml}$ poly-D-lysine and $4 \mu \mathrm{g} / \mathrm{ml}$ laminin at a density of 5 hemispheres/plate $\left(\sim 4 \times 10^{5}\right.$ cells/culture well) in MEM (Earle's salts) supplemented with $5 \%$ horse serum, $5 \%$ fetal bovine serum, and $21 \mathrm{mM}$ glucose. Proliferation of non-neuronal cells was halted by inclusion of $10 \mu \mathrm{M}$ cytosine arabinoside at days in vitro (DIV) 7 when astrocytes became confluent underneath neurons. Cultures were fed with growth medium identical to the plating medium, but lacking fetal serum 2-3 days later. After this, cultures were fed with growth medium twice a week and maintained at $37^{\circ} \mathrm{C}$ in a humidified $\mathrm{CO}_{2}$ incubator $(5 \%)$ until the time of the experiments. Animal care and treatment were in compliance with a protocol approved by our institutional animal care committee.

Injury paradigms: To induce free radical injury, cortical cell cultures (DIV 12) were exposed continuously to $50 \mu \mathrm{M}$ $\mathrm{Fe}^{2+}$ or $150 \mu \mathrm{M}$ BSO for $24 \mathrm{~h}$. $\mathrm{Fe}^{2+}$ produces hydroxyl radicals via a Fenton reaction [16], and BSO, a $\gamma$-glutamyl- 
cysteine synthase inhibitor, induces radical injury by depleting glutathione in the cell. Cortical cell cultures (DIV 12) were exposed for $24 \mathrm{~h}$ to $30 \mu \mathrm{M}$ NMDA to induce excitotoxicity, and $100 \mathrm{nM}$ staurosporine, a non-selective protein kinase inhibitor, to induce apoptosis [17].

Analysis of neuronal death: The morphological changes of cells were observed under a phase-contrast microscope. To quantify the neuronal injury, lactate dehydrogenase $(\mathrm{LDH})$ release into bathing media was analyzed $24 \mathrm{~h}$ later [18]. The LDH values were compared to the mean LDH values released after $24 \mathrm{~h}$ exposure to sham wash $(0 \%$ death) and $500 \mu \mathrm{M}$ NMDA (100\% death).

Assay of radical scavenging activity: $\mathrm{DPPH}$, a stable free radical, was dissolved in ethanol. 5-HT and other materials of interest were added to $100 \mu \mathrm{M}$ DPPH for $10 \mathrm{~min}$. The radical scavenging activity was determined by measuring the decrease in levels of DPPH at $517 \mathrm{~nm}$ [19].

\section{RESULTS}

5-HT attenuates free radical-induced neuronal death: We examined whether 5-HT may influence neuronal death induced by oxidative stress. Cultures (DIV 12) that were exposed to $50 \mu \mathrm{M} \mathrm{FeCl}_{2}$ or $150 \mu \mathrm{M}$ BSO showed cell body swelling within $8 \mathrm{~h}$ and underwent widespread neuronal death $24 \mathrm{~h}$ later (Fig. 1a). Co-treatment with 5-HT protected the neurons from free radical-induced cell body swelling as well as death in a dose-dependent manner (Fig. 1a,b).

The anti-oxidant effects of 5-HT are not mediated through
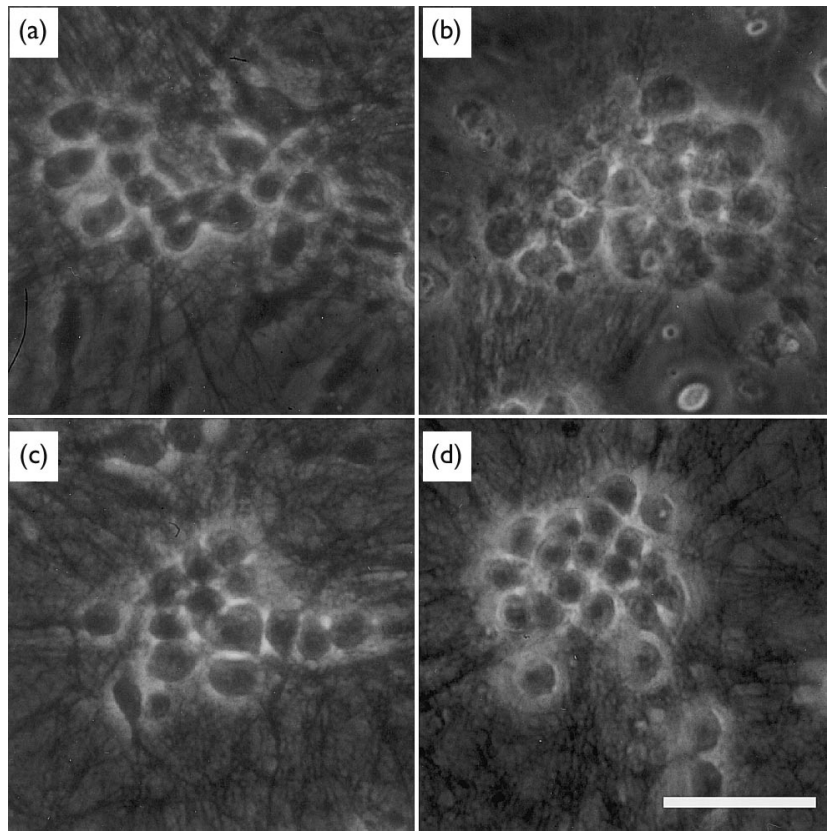

5-HT receptors: To determine the type of the receptor activation involved in the anti-oxidative effects of 5-HT, cortical cell cultures (DIV 12) were exposed to $50 \mu \mathrm{M} \mathrm{Fe}{ }^{2+}$ alone or with various 5-HT receptor antagonists for $24 \mathrm{~h}$. Five antagonists of 5 -HT were used in this experiment; $50 \mu \mathrm{M}$ pindolol $\left(5-\mathrm{HT}_{1 \mathrm{~A} / 1 \mathrm{~B}}\right.$ receptor antagonist), $10 \mu \mathrm{M}$ cyproheptadine hydrochloride $\left(5-\mathrm{HT}_{2}\right.$ nonselective antagonist), $\quad 30 \mu \mathrm{M}$ propisetron (selective $5-\mathrm{HT}_{3}$ receptor antagonist), $20 \mu \mathrm{M}$ RS 39604 hydrochloride (selective $5-\mathrm{HT}_{4}$ receptor antagonist) or $5 \mu \mathrm{M}$ dihydroxyergocristine mesylate (non-selective 5-HT receptor antagonist). We used each antagonist at maximal nontoxic concentrations. No antagonists reversed the protective effect of $5-\mathrm{HT}$ on $\mathrm{Fe}^{2+}$-induced necrosis (Fig. 2). These data suggest that the protective effect of 5-HT against free radical injury on cortical neurons is not through receptor activation.

5-HT has direct antioxidant effects: Failure of receptor subtype-specific antagonists to reverse the protective effect raised the possibility of direct free radical scavenging activity of 5-HT. Therefore we tested the possibility of 5$\mathrm{HT}$ as a direct antioxidant using the DPPH assay. Surprisingly, 5-HT, like trolox, a vitamin E analogue, substantially lowered levels of DPPH, a stable free radical-forming agent, in a cell-free system (Table 1). $\alpha$-Methyl-5-HT, which has been reported to increase neuronal survival, also showed free radical scavenging activity [7] (Table 1).

5-HT has no protective effects on excitotoxic necrosis or staurosporine-induced apoptosis: Activation of NMDA receptors induces a necrotic type of neuronal injury in

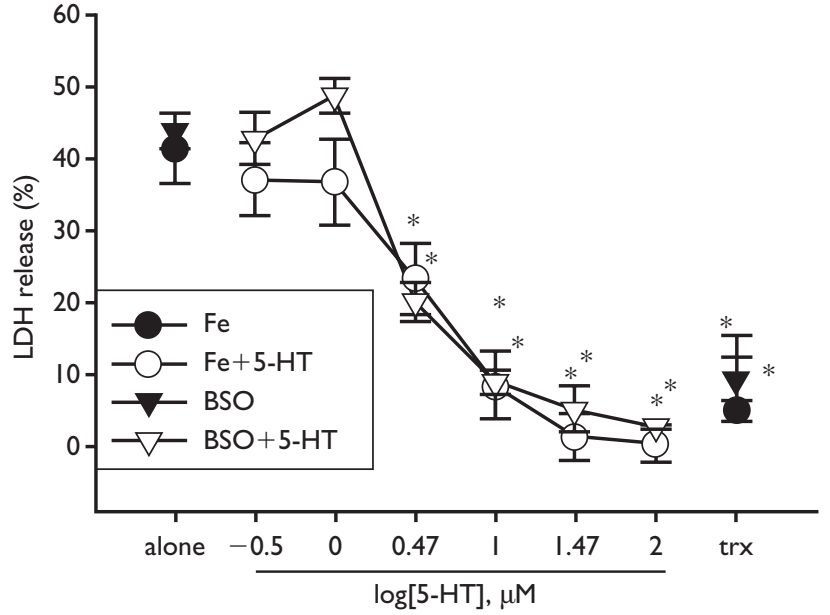

Fig. I. Attenuation by 5-HT of free radical-induced neurotoxicity. (a) Phase-contrast micrographs of cultured cortical neurons (DIV I2) taken after $14 \mathrm{~h}$ exposure to a sham control (a), $50 \mu \mathrm{M} \mathrm{FeCl}_{2}$ (b) $50 \mu \mathrm{M} \mathrm{FeCl}$ plus $30 \mu \mathrm{M} 5-\mathrm{HT}$ (c), and $50 \mu \mathrm{M} \mathrm{FeCl}$ plus $100 \mu \mathrm{M}$ trolox (d). Note the swelling of neuronal bodies that is prevented with $5-\mathrm{HT}$ or trolox. Bar $=50 \mu \mathrm{m}$. (b) Cortical cultures were exposed to $50 \mu \mathrm{M} \mathrm{FeCl}_{2}(\mathrm{closed}$ circle) or I50 $\mu \mathrm{M}$ BSO alone (closed triangle) or with the indicated dose of 5- $\mathrm{HT}$ (open triangle). For a positive control, $100 \mu \mathrm{M}$ trolox (trx) was added with $\mathrm{FeCl} 2$ or $\mathrm{BSO}$. $\mathrm{LDH}$ in the bathing media was measured $24 \mathrm{~h}$ later. Values are means \pm s.e.m. $(n=8$ culture wells per condition), expressed as a percentage of the mean LDH value corresponding to the near-complete neuronal death induced by $24 \mathrm{~h}$ exposure to $500 \mu \mathrm{M}$ NDA (=100). *Significant difference from relevant control $\left(50 \mu \mathrm{M} \mathrm{Fe}^{2+}\right.$ or $150 \mu \mathrm{M}$ BSO alone), $p<0.05$ using ANOVA and Student-Newman-Keuls' test. 




Fig. 2. Antagonists of 5-HT receptors do not reverse the anti-oxidant effects of 5-HT. Mixed cortical cell cultures (DIV I2) were exposed to $50 \mu \mathrm{M} \mathrm{Fe}{ }^{2+}$ alone $(\mathrm{Fe})$ or with the following additions: $30 \mu \mathrm{M} 5-\mathrm{HT}$ $(\mathrm{Fe}+5-\mathrm{HT}), 5-\mathrm{HT}$ plus one of the antagonists $(\mathrm{Fe}+5-\mathrm{HT}+\mathrm{ant})(10 \mu \mathrm{M}$ cyproheptadine hydrochloride (CPH), $5 \mu \mathrm{M}$ dihydroxyergocristine mesylate (DHE), $50 \mu \mathrm{M}$ pindolol (PND), $20 \mu \mathrm{M}$ RS 39604 hydrochloride (RS) or $30 \mu \mathrm{M}$ propisetron (PPS)), or to antagonists only (Fe+ant). Also, toxicity of the antagonists only was examined (ant). Neuronal death was analyzed $24 \mathrm{~h}$ later by measurement of LDH in the bathing media, with values expressed as means \pm s.e.m. ( $n=8$ culture wells per condition). *Significant difference from $\mathrm{Fe}^{2+}$ alone (CTRL), at $p<0.05$ using ANOVA and Student-Newman-Keuls' test.

Table I. 5-HT acts as a direct antioxidant.

\begin{tabular}{lcl}
\hline Treatment & Concentration $(\mu \mathrm{M})$ & $\begin{array}{l}\mathrm{A}_{517 \mathrm{~nm}} \text { decrease } \\
(n=6)\end{array}$ \\
\hline Serotonin & 0.3 & $0.3265 \pm 0.0180^{*}$ \\
& 1 & $0.3560 \pm 0.0207^{*}$ \\
& 3 & $0.4765 \pm 0.0167^{*}$ \\
& 10 & $0.7572 \pm 0.0234^{*}$ \\
a-Methyl-5-HT & 10 & $1.0207 \pm 0.0067^{*}$ \\
Trolox & 10 & $1.0525 \pm 0.0053^{*}$ \\
& 100 & $0.4775 \pm 0.0398^{*}$ \\
\hline
\end{tabular}

The free radical scavenging activity of $5-\mathrm{HT}, \alpha$-methyl $5-\mathrm{HT}$ or trolox was determined by measuring the decrease in the stable free radical DPPH at $A_{517}$ after a $10 \mathrm{~min}$ reaction with $0.1 \mathrm{mM}$ DPPH; values are expressed as means \pm s.e.m. ( $n=4$ for condition). * Significant difference from DPPH alone, at $p<0.05$ using ANOVA and Student-Newman-Keuls' test.

cortical cell cultures characterized by cell body swelling and reversed by NMDA receptor antagonists such as MK$801(30 \mu \mathrm{M})$. 5-HT has no significant effect on NMDAinduced neuronal necrotic death (Fig. 3).

Staurosporine-induced apoptosis of neurons is accompanied by cell body shrinkage and chromatin condensation and attenuated by a protein synthesis inhibitor, cycloheximide $(1 \mu \mathrm{g} / \mathrm{ml})$. Similar to the situation with NMDAinduced necrosis (Fig. 3), 5-HT has no effects on $100 \mathrm{nM}$ staurosporine-induced neuronal apoptosis.

\section{DISCUSSION}

Due to its wide distribution throughout the cortex, the

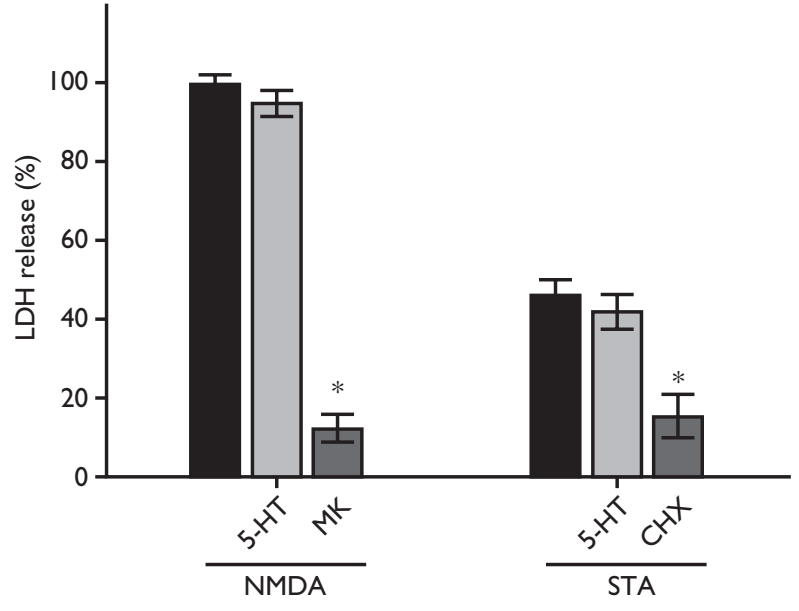

Fig. 3. 5-HT has no effects on excitotoxicity or staurosporine- induced neuronal apoptosis. Sister cultures (DIV I2) were exposed to $30 \mu \mathrm{M}$ NMDA (NMDA), $100 \mathrm{nM}$ staurosporine (STA) alone or with $30 \mu \mathrm{M} 5-\mathrm{HT}$ or $10 \mu \mathrm{M}$ MK-80I (MK) or I $\mu \mathrm{g} / \mathrm{ml}$ cycloheximide (CHX). Neuronal death was analyzed $24 \mathrm{~h}$ later by measurement of LDH in the bathing media. Values are means \pm s.e.m. $(n=8$ culture wells per condition). *Significant difference from NMDA or staurosporine alone (CTRL), at $p<0.05$ using ANOVA and Student-Newman-Keuls' test.

serotonergic system has been proposed to be in a position of regulating neuronal survival [20]. Here we used a dissociated cortical cell culture system to investigate the role of 5-HT on well-defined neuronal death paradigms. It was found that 5-HT prevented free radical inducednecrotic death of neurons in mixed cortical cultures. This protective effect was not reversed with receptor subtypespecific antagonists, therefore implying a non-receptormediated neuroprotective action of 5-HT. Our findings suggested strongly that 5 -HT act as a direct free radical scavenger. The ineffectiveness of 5-HT on excitotoxicity or staurosporine-induced apoptosis also suggests the specificity of 5-HT on free radical mediated neurotoxicity.

Recently, evidence indicating receptor subtype specificity in the survival-enhancing effects of the serotonergic system has appeared. In cerebral cortical culture, addition of 5-HT or its $2 \mathrm{~A} / 2 \mathrm{C}$ receptor agonist $\alpha$-methyl-5-HT in the culture medium promote the survival of glutamatergic neurons [7]. In C6 glioma cell cultures, 5-HT protects against gliotoxicity produced by high doses (millimolar concentration) of glutamate, which induce free radical injury through the blockade of cystine uptake [8]. A highly potent and selective $5-\mathrm{HT}_{1 \mathrm{~A}}$ receptor agonist, Bay X $3702((-)-(\mathrm{R})-2-[4-[[(3,4-$ dihydro-2H-1-benzopyran-2-yl)methyl]amino]butyl]-1,2,benzisothiazol-3(2H)-one 1,1-dioxide monohydrochloride), prevents the damage induced by the excitotoxin glutamate, serum deprivation or staurosporine-induced apoptosis in rat hippocampal cultures or chick embryonic neuron culture [9,11-13]. It also prevents ischemic insults produced by permanent occlusion of the middle cerebral artery in rats with cerebral artery occlusion [10]. These results suggest that $5-\mathrm{HT}_{1 \mathrm{~A}}$ or $5-\mathrm{HT}_{2 \mathrm{~A} / 2 \mathrm{C}}$ receptors play an important role in the protective action of 5-HT. However, in our experiments, pindolol $\left(5-\mathrm{HT}_{1 \mathrm{~A} / 1 \mathrm{~B}}\right.$ receptor antagonist) or cyproheptadine (5- $\mathrm{HT}_{2}$ non-selective antagonist) did not inhibit the neuroprotective action of 5-HT. Given the similarity of 
chemical structure between 5-HT and $\alpha$-methyl-5-HT, this apparent discrepancy may be explained in the light of our data indicating that both compounds possess the ability to reduce the stable free radical DPPH (Table 1).

As in the case of growth factors which prevent the apoptotic type of neuronal injury but potentiate NMDA- or free radical-induced neuronal injury [21,22], defining the specificity of neuroprotective maneuvers on neuronal injury were important to develop an adequate strategy of neuroprotection. Our results show that the protective effect of $5-\mathrm{HT}$ is confined to free radical-induced neuronal injury. These findings are compatible with a previous report that 5-HT prevents free radical injury induced by high doses of glutamate in G6 glioma cells but it does not prevent the excitoxicity of glutamate in cortical or cerebellar neuronal culture [8].

As knowledge about the pathological role of free radical injury in neurodegenerative systems has evolved, considerable effort has been focused on clarifying the mechanism of anti-oxidative strategies. Therefore, the purpose of our study was to clarify the mechanism of neuroprotection of 5-HT on neuronal injury. Antioxidant mechanisms can be divided into two major categories, i.e. direct antioxidant effects or enhancement or turning on of innate antioxidative defense mechanisms. In contrast to previous proposals, our findings suggest a direct antioxidant property of 5-HT which is also compatable with previous findings that showed the phenolic moiety possesses the radical scavenging activity [23].

From these results, 5-HT can be considered not only as endogeneous neurotransmitter, but also as an antioxidant defense mechanism in the central nervous system.

\section{CONCLUSION}

Our results provide evidence that 5-HT attenuates free radical induced neuronal death without being coupled to 5 -HT receptors in cultured mouse cortical neurons. Moreover, 5-HT possesses a direct antioxidant property. In addition, 5-HT has no modulating effects on excitotoxicity or staurosporine-induced apoptosis. This neuroprotective property suggests a dual role for 5-HT as an anti-oxidative defense mechanism and neurotransmitter.

\section{REFERENCES}

1. Choi DW, Koh JY and Peters S. J Neurosci 8, 185-196 (1988).

2. Koh JY, Goldberg MP, Hartley DM et al. J Neurosci 10, 693-705 (1990).

3. Buisson A and Choi DW. Neuropharmacology 34, 1081-1087 (1995).

4. Noh JS, Kim EY, Kang JS et al. Exp Neurol 159, 217-224 (1999).

5. Tork I. Ann N Y Acad Sci 600, 9-34 (1990).

6. Roth BL. Ann Clin Psychiatry 6, 67-78 (1994).

7. Dooley AE, Pappas IS and Parnavelas JG. Exp Neurol 148, 205-214 (1997).

8. Shinagawa S. Neuroscience 59, 1043-1050 (1994).

9. Suchanek B, Struppeck H and Fahrig T. Eur J Pharmacol 355, 95-101 (1998).

10. Semkova I, Wolz P and Krieglstein J. Eur J Pharmacol 359, 251-260 (1998).

11. Ahlemeyer B and Krieglstein J. Brain Res 777, 179-186 (1997).

12. Ahlemeyer B, Glaser A, Schaper C et al. Eur J Pharmacol 370, 211-216 (1999).

13. Ahlemeyer B, Beier H, Semkova I et al. Brain Res 858, 121-128 (2000).

14. Zilkha-Falb R, Ziv I, Nardi N et al. Cell Mol Neurobiol 17, 101-118 (1997).

15. Noh JS and Gwag BJ. Exp Neurol 146, 604-608 (1997).

16. Halliwell B and Gutteridge JM. FEBS Lett 307, 108-112 (1992).

17. Koh JY, Wie MB, Gwag BJ et al. Exp Neurol 135, 153-159 (1995).

18. Koh JY and Choi DW. J Neurosci Methods 20, 83-90 (1987).

19. Cotelle N, Bernier JL, Catteau JP et al. Free Radicals Biol Med 20, 35-43 (1996).

20. Hanley MR. Nature 340, 97-98 (1989).

21. Koh JY, Gwag BJ, Lobner D et al. Science 268, 573-575 (1995).

22. Gwag BJ, Koh JY, Chen MM et al. Neuroreport 7, 93-96 (1995).

23. Moosmann B and Behl C. Proc Natl Acad Sci USA 96, 8867-8872 (1999).

Acknowledgements: This study was supported by Korea Science and Engineering Foundation Core research program grant 981 07I 3-092-2 (J.S.N.). 